\title{
ARTIFACTS AND ERRORS IN EBSD MAPPING OF RETAINED AUSTENITE IN TRIP STEEL
}

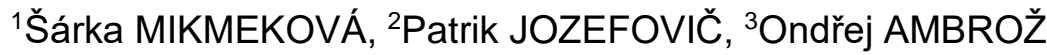 \\ ${ }^{1}$ Institute of Scientific Instruments of the CAS, Brno, Czech Republic, EU, \\ sarkaj@isibrno.cz,patjoz@isibrno.cz,ondrei@isibrno.cz
}

https://doi.org/10.37904/metal.2021.4160

\begin{abstract}
The present work aims to demonstrate artifacts and errors in visualization of retained austenite phase in TRIP steel by an electron back-scattered diffraction (EBSD) technique. Retained austenite phases size and shape obtained by the EBSD are directly compared with a real image of these phases acquired by means of an atomic force microscopy (AFM). The effect of the step size parameter used for the EBSD analysis on the retained austenite phase fraction and morphology is discussed in detail and quantified. Surface roughness as a barrier for the imaging of fine features situated on a specimen surface is demonstrated.
\end{abstract}

Keywords: EBSD, SEM, AFM, retained austenite, TRIP steel

\section{INTRODUCTION}

Transformation induced plasticity (TRIP) steels exhibit excellent mechanical properties, such as high strength and ductility, due to their unique multi-phase structure [1]. Correct understanding of the structure-property relationship is conditioned by existence of an adequate characterization technique enabling visualization of the phases with high spatial resolution, their clear identification, and possibility to investigate large area on a specimen surface. Retained austenite plays a key role in determining the mechanical properties of TRIP steels and accurate knowledge of its fraction, size, morphology, and distribution with a matrix is crucial for further development of advanced high strength steels (AHSS). An Electron Back-Scattered Diffraction (EBSD) is the most commonly used technique for visualization of the retained austenite phase in TRIP steels due to clean difference between the Kikuchi patterns of the austenite FCC phase and other BCC phases (ferrite, bainite, martensite. However, the EBSD technique has a limited spatial resolution (around $40 \mathrm{~nm}$ ) and requires perfect specimen surface without any contamination and preparation artifacts [2,3]. Modern AHSS steels contain nano-sized retained austenite phases which become for the conventional EBSD invisible. The next problem is a real specimen surface. Standardly used sample preparation technique for the EBSD is an electropolishing. TRIP steels consist of several phases and each of them has its own etching rate in an electrolyte solution. It results in a characteristic surface topography where the secondary phases arise from the matrix. The EBSD data are typically acquired at high stage tilt (about $70^{\circ}$ ) and slow scan speed. It leads to tilt and drift distortions that obscure or deform the phases in the final EBSD maps. In this work, we demonstrate artifacts and errors in the EBSD mapping of the retained austenite phase in a TRIP steel.

\section{EXPERIMENTAL}

\subsection{Instruments and techniques (AFM, SEM, EBSD)}

Several characterization techniques were utilized for sample analysis. The surface morphology was investigated by an atomic force microscopy (AFM) integrated to a scanning electron microscope (SEM), namely by a LiteScope ${ }^{T M}$ (NenoVision) [4]. The LiteScope ${ }^{T M}$ instrument enables simultaneous AFM and SEM 
data acquisition and their accurate correlation. The UHR SEM Magellan $400 \mathrm{~L}$ (Thermofisfer Scientific [5]) equipped with the Hikari EBSD camera from EDAX were employed for EBSD analysis [6]. The EDAX Team software was used to acquire the data under various experimental conditions. The EBSD data were analyzed using OIM Analysys ${ }^{\mathrm{TM}} 7$ software (EDAX). The EBSD parameters used in this study are following: accelerating voltage: $20 \mathrm{kV}$, beam current: 6,4 nA, working distance: $8 \mathrm{~mm}$, binning: $4 \times 4$.

\subsection{Specimen}

A specimen under investigation was a TRIP steel $(0.2 \mathrm{C}-1.5 \mathrm{Si}-2 \mathrm{Mn})$ containing $12 \%$ of retained austenite phase (measured by an X-Ray diffraction technique). The specimen was prepared by a conventional metallographic technique, i.e. mechanically polished with the final step of $0.25 \mu \mathrm{m}$ diamond paste. As the final step of the specimen preparation, a conventional electro-polishing technique was applied. Figure 1 shows a result of correlative probe and electron microscopy (CPEM) technique, namely the SEM secondary electron (SE) image + AFM map of the specimen surface. As is visible, the specimen surface is relatively smooth, scratch-free, and without any preparation artifacts and contaminations. The secondary phases, i.e. the martensite and the retained austenite, arise from the matrix. It is a consequence of different etching rate of these phases in the electrolyte solution.

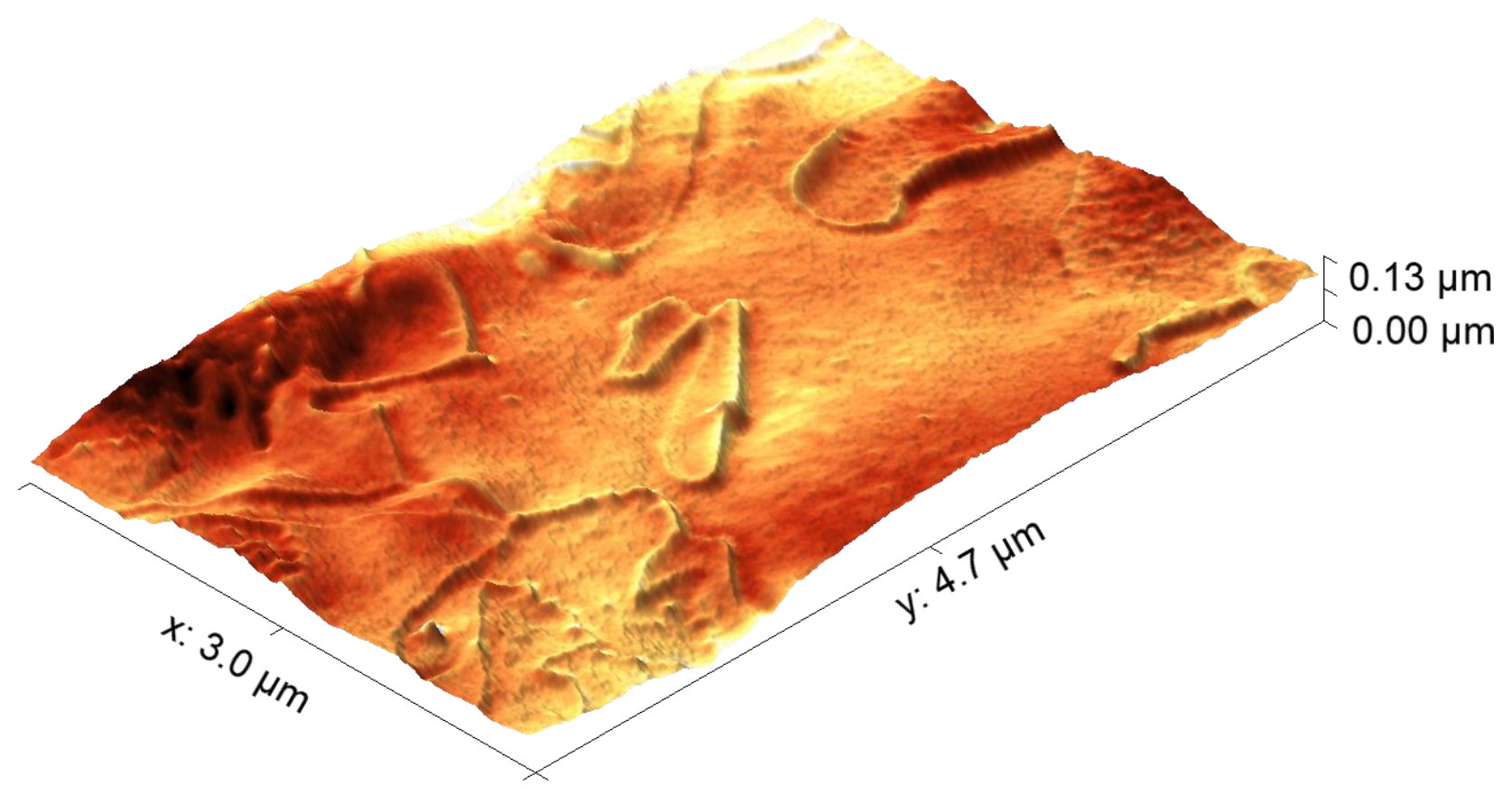

Figure $1 \mathrm{AFM}+$ SEM SE image of the TRIP steel specimen after conventional electro- polishing process.

\section{RESULTS AND DISCUSSION}

Figure 2 shows the EBSD phase + image quality (IQ) maps of the same field of view obtained using different step size presenting information about distribution and morphology of the retained austenite phases in the TRIP steel specimen. Other EBSD conditions were fixed (i.e. identical scan area $33 \times 26 \mu \mathrm{m}$ and camera settings). Obviously, the EBSD maps acquired with small step size are able to detect also fine austenite phases. Utilizing of coarser step sizes leads to neglecting of fine phases and significant reduction of area fraction of the retained austenite. The step size significantly affects the acquisition time of the EBSD maps. The total size of the analysed area is $875 \mu \mathrm{m}^{2}$ and the acquisition time for the EBSD phase maps in Figure 2 are as follows: $2 \mathrm{~h} 7 \mathrm{~m} \mathrm{13s} \mathrm{(40} \mathrm{nm} \mathrm{step} \mathrm{size),} 58 \mathrm{~m} 20 \mathrm{~s}$ ( $60 \mathrm{~nm}$ step size), $33 \mathrm{~m} 22 \mathrm{~s}$ ( $80 \mathrm{~nm}$ step size), $21 \mathrm{~m} 3 \mathrm{~s}$ 
(100 nm step size), $9 \mathrm{~m}$ 19s ( $50 \mathrm{~nm}$ step size), $5 \mathrm{~m}$ 18s (200 nm step size), $2 \mathrm{~m}$ 24s ( $300 \mathrm{~nm}$ step size), $1 \mathrm{~m}$ $21 \mathrm{~s}$ ( $400 \mathrm{~nm}$ step size), and $48 \mathrm{~s}$ (500 $\mathrm{nm}$ step size).
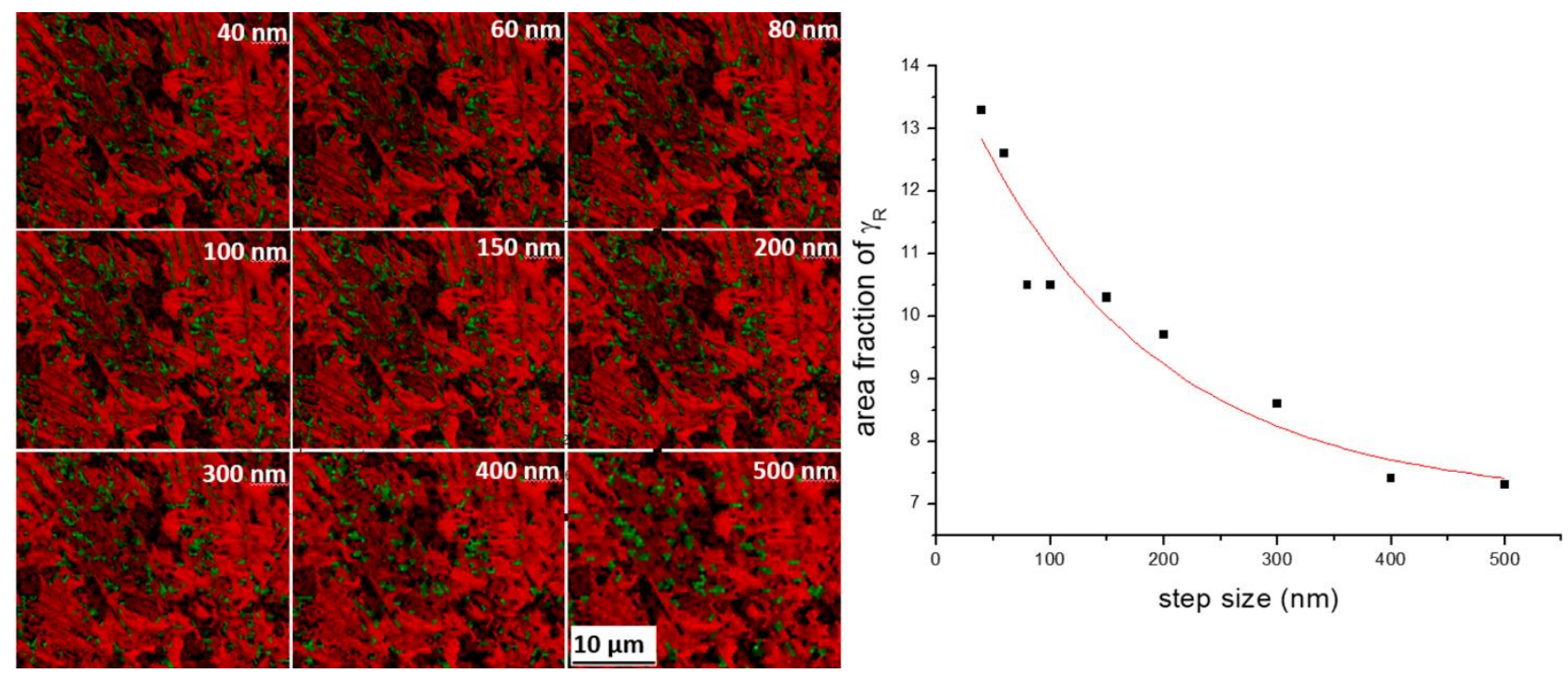

Figure 2 EBSD phase + image quality maps of the identical area on the TRIP steel surface collected with various step size: $40 \mathrm{~nm}, 60 \mathrm{~nm}, 80 \mathrm{~nm}, 100 \mathrm{~nm}, 150 \mathrm{~nm}, 200 \mathrm{~nm}, 300 \mathrm{~nm}, 400 \mathrm{~nm}$, and $500 \mathrm{~nm}$ (as marked in the maps), together with a corresponding graph demonstrating the effect of the step size on the area fraction of retained austenite.

The EBSD technique offers not only quantitative but also qualitative information about the retained austenite phase. Austenite size and shape are also important factor affecting the mechanical properties. Figure 3 shows a series of the EBSD phase maps of the same point of view obtained with different step size. The AFM topography map on the right side presents a reference image of the same area and shows a real shape and size of the retained austenite phase. Obviously, the austenite phase in the EBSD maps looks different in comparison with the AFM map. The elongated shape is a consequence of the specimen drift. Moreover, the EBSD data miss fine details even using extremely small step size. As visible in the AFM image, the austenite phase arises from the matrix, which combined with a high specimen tilt $\left(75^{\circ}\right)$ results in errors in the EBSD imaging. Direct comparison of the EBSD and the AFM data is shown in Figure 4.

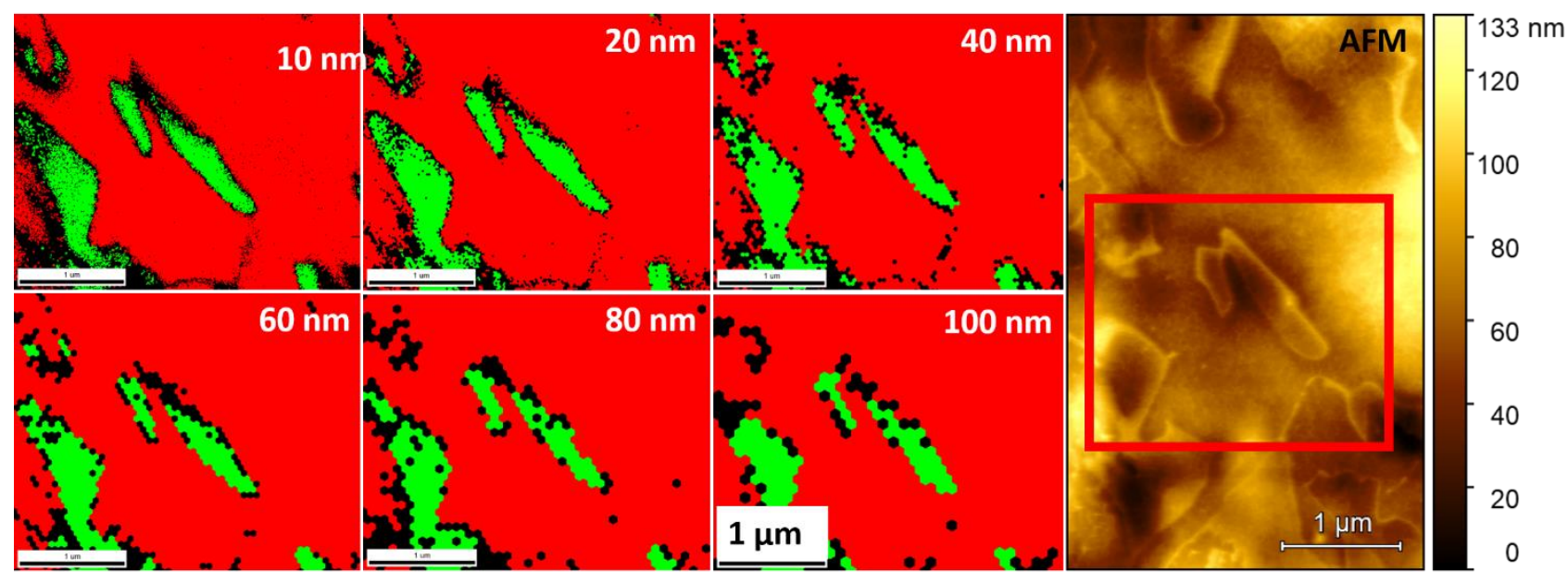

Figure 3 Effect of the step size on the shape of retained austenite. AFM surface topography map shows the real shape of the austenite phase (on the right site). 


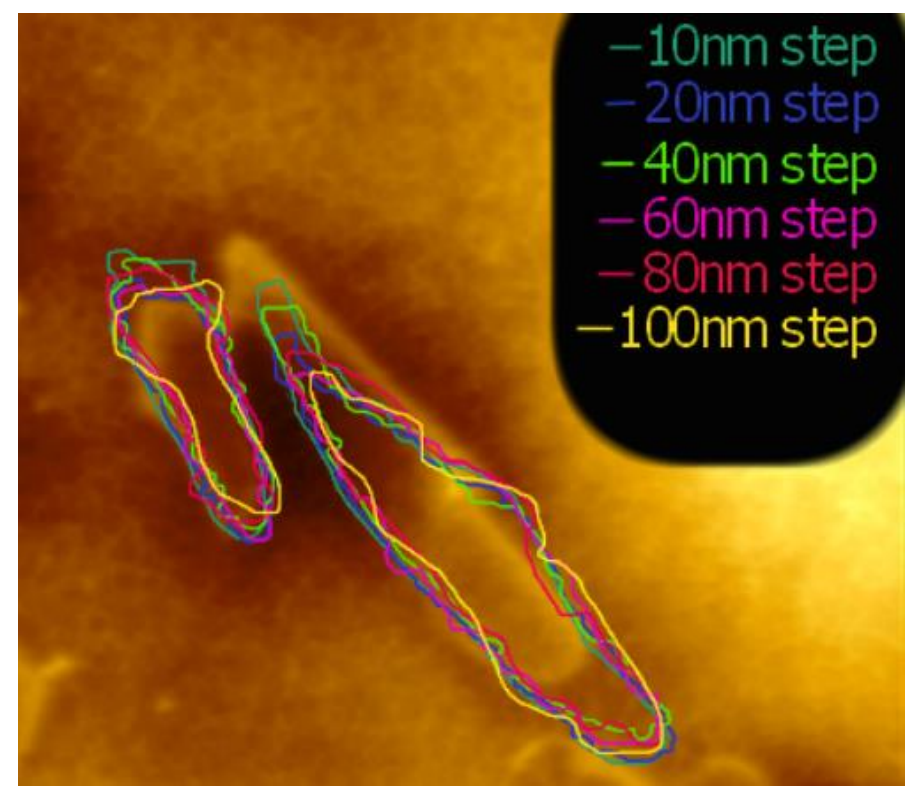

\begin{tabular}{|c|c|c|c|}
\hline Step & $\begin{array}{c}\text { Area } \\
\text { [um2] }\end{array}$ & $\begin{array}{l}\text { difference vs } \\
\text { reality [ } \nu \mathrm{m} 2]\end{array}$ & $\%$ diff \\
\hline AFM & 0.458 & & \\
\hline $10 \mathrm{~nm}$ & 0.499 & $-0,041$ & 8.95 \\
\hline $20 \mathrm{~nm}$ & 0.386 & 0.072 & 15.72 \\
\hline $40 \mathrm{~nm}$ & 0.362 & 0.096 & 20.96 \\
\hline $60 \mathrm{~nm}$ & 0.338 & 0.12 & 26.20 \\
\hline $80 \mathrm{~nm}$ & 0.342 & 0.116 & 25.33 \\
\hline $100 \mathrm{~nm}$ & 0.303 & 0.155 & 3384 \\
\hline
\end{tabular}

Figure 4 AFM surface topography map overlapped by the austenite phase contour obtained from the EBSD maps collected with various step size, together with the corresponding table demonstrating difference between the real size of the retained austenite constituent and size of the same phase obtained from the EBSD phase maps.
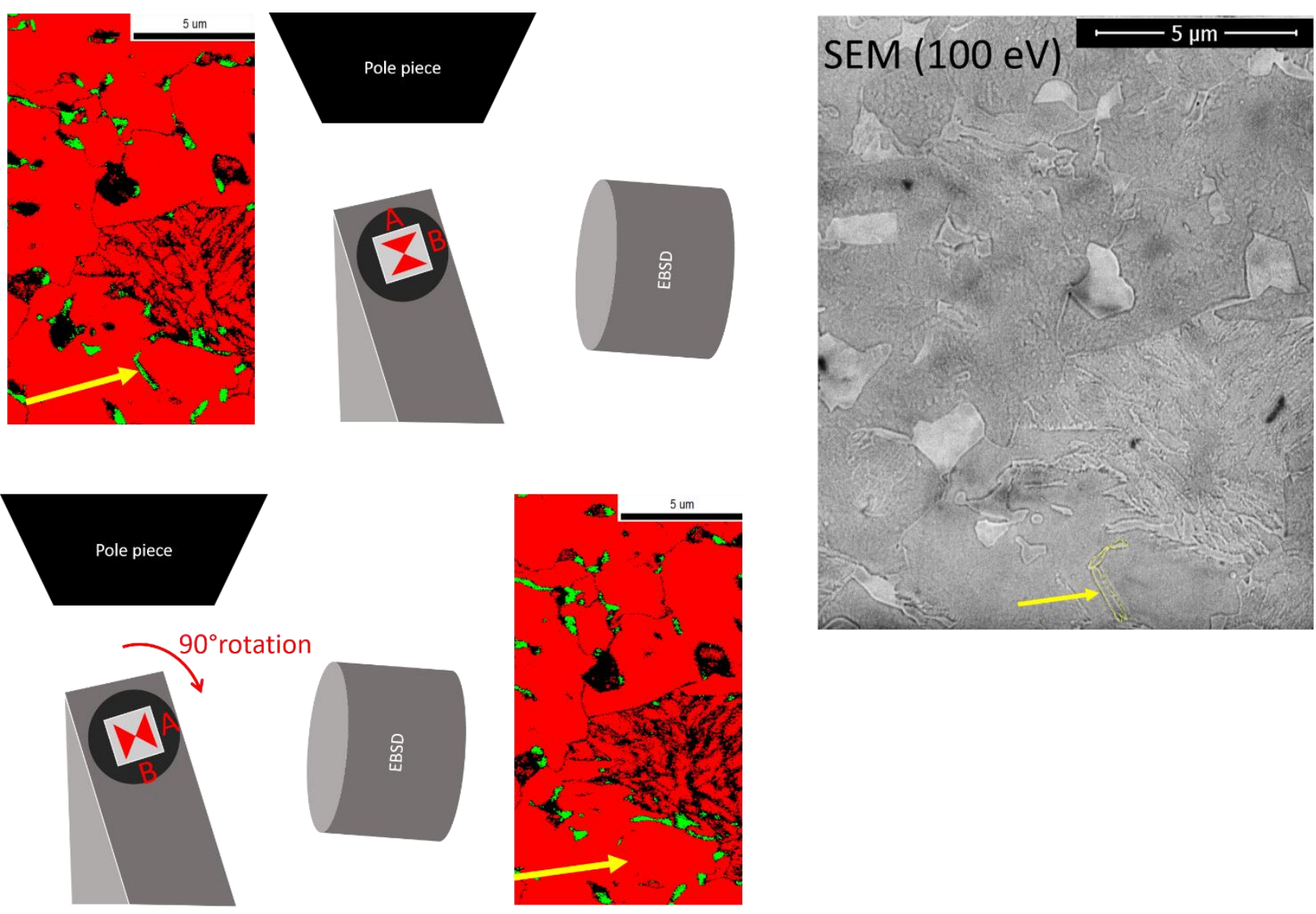

Figure 5 EBSD phase maps of the identical area on the TRIP steel surface collected using different specimen position on the stage (i.e. specimen rotation $0^{\circ}$ and $90^{\circ}$ ), together with corresponding SEM micrograph obtained at $100 \mathrm{eV}$ landing energy of the primary electrons. 
Figure 5 demonstrates the effect of the experimental setting on a visibility of the secondary phases. As mentioned above, the secondary phase have slower etching rate in the electrolytic solution in comparison with the ferrite matrix and arises from the surface. It results in shading of features situated in unsuitable positions. The yellow arrow in the EBSD maps and in the SEM micrograph indicates a position of the selected retained austenite phase. Only change of the specimen position on the stage, i.e. the specimen is rotated to $90^{\circ}$, leads to different results of the austenite mapping by the EBSD.

\section{CONCLUSION}

The EBSD technique is a common tool for characterization of retained austenite in multiphase steels. In this paper artifacts and errors in the EBSD mapping of the retained austenite in the TRIP steel are demonstrated. The main results of the paper are summarized below.

- $\quad$ Precise mapping of the retained austenite phase in modern TRIP steels requires very fine step size.

- $\quad$ The EBSD phase maps are not showing a real shape and size of the retained austenite phase.

- $\quad$ Selective etching rate of the secondary phases in TRIP steels during the electropolishing process results in their arising from the matrix. It leads to invisibility of some phases situated in unsuitable positions.

\section{ACKNOWLEDGEMENTS}

\section{The research was supported by Technology Agency of the Czech Republic - National Centre of Competence No. TN01000008.}

\section{REFERENCES}

[1] www.worldautosteel.org

[2] ZHANG, Y., LAI, P., JIA, H., XINHUA, J., CUI, G. Investigation of test parameters on EBSD analysis of retained austenite in TRIP and pipeline steels. Metals. 2019, vol. 9, no. 1, pp. 94-100.

[3] MAN, O., PANTELEJEV, L., PESINA, Z. EBSD analysis of phase composition of TRIP steel on various strain levels. Materials Engineering. 2009, vol. 16, no. 2, pp. 15-21.

[4] www.nenovision.com

[5] UHR SEM Magellan user operation manual (Thermofisher Scientific)

[6] www.edax.com 